\title{
The effect of partial reinforcement on the acquisition and extinction of sign-tracking and goal-tracking in the rat
}

\author{
GRAHAM C. L. DAVEY and GARY G. CLELAND \\ City University, London ECIVOHB, England
}

\begin{abstract}
The acquisition and extinction of sign-tracking (CS contact) and goal-tracking (food-site approach) responses in rats were studied under partial (50\%) and continuous (100\%) Pavlovian appetitive reinforcement schedules. Using a retractable lever as the CS for food, partial reinforcement during acquisition training produced a higher asymptotic rate of sign-tracking than continuous reinforcement, whereas continuous reinforcement developed a higher asymptotic level of goal-tracking during CS presentation. However, extinction following partial reinforcement produced a resistance to extinction of goal-tracking but not of sign-tracking. This result suggests that although goal-tracking and sign-tracking are both discriminated responses to the CS, they possess some different dynamic characteristics that possibly indicate sensitivity to different controlling variables.
\end{abstract}

Procedures that pair a localizable conditioned stimulus (CS) with an appetitive reinforcer (autoshaping) generate two apparently conflicting response tendencies in the rat: Rats will approach either the signal for food (sign-tracking) or the food tray itself (goal-tracking). Boakes $(1977,1979)$ found that the frequency of both responses increased as autoshaping training progressed, and both responses decreased in frequency when food was removed (extinction).

For some years now, the stimulus correlations that generate and maintain these behaviors have been a source of intensive debate, and the current consensus is that sign-tracking obeys Pavlovian rather than instrumental associative rules (e.g., Hearst, 1979). Support for this viewpoint accrues from the fact that sign-tracking (1) has been shown to be a function of the duration of the intertrial interval (Gibbon, Baldock, Locurto, Gold, \& Terrace, 1977; Terrace, Gibbon, Farrell, \& Baldock, 1975), (2) can be suppressed by the introduction of unsignaled reinforcer deliveries during the intertrial interval (Gamzu \& Williams, 1971, 1973), and (3) is eliminated only with great difficulty following the imposition of an omission contingency (Atnip, 1977; Schwartz \& Williams, 1972; Williams \& Williams, 1969). All of these effects are typically found using more traditional Pavlovian responses.

However, the status of goal-tracking is less welldefined. While, like sign-tracking, it occurs during CS presentation, it is a response that bears a more intimate relation to reinforcer presentation: Since it is by necessity a response that has to precede reinforcer receipt, it may be more directly influenced by instrumental responsereinforcer correlations than sign-tracking is. While Trust.

Gary Cleland is in receipt of a scholarship from the Wellcome

studies that have attempted to investigate control of goal-tracking by instrumental correlations have been largely inconclusive, they have favored instrumental rather than Pavlovian interpretations (Farwell \& Ayres, 1979; Weardon, in press).

The present study is designed to supplement the evidence identifying the correlations influencing signtracking and goal-tracking by investigating the effect of partial reinforcement on the extinction of the two responses. Increased resistance to extinction following partial reinforcement is a robust and reliable phenomenon in the instrumental literature (cf. Lewis, 1960; Mackintosh, 1974, pp. 431-467) and might be expected to be exhibited by goal-tracking if it is a response that is readily comparable to more traditional instrumental responses. However, the partial reinforcement extinction effect (PREE) is a more elusive phenomenon in Pavlovian conditioning and might not be exhibited by sign-tracking in the rat (but see Gibbon, Farrell, Locurto, Duncan, \& Terrace, 1980).

\section{METHOD}

Subjects

The subjects were 14 male hooded Lister rats approximately 90 days old at the outset of the experiment. All were experimentally naive and maintained at approximately $80 \%$ of their free-feeding body weights throughout the experiment.

\section{Apparatus}

The experimental chambers consisted of purpose-built Skinner boxes marketed by Campden Instruments Limited. The internal dimensions of the boxes were $20.5 \mathrm{~cm}$ high, $23.5 \mathrm{~cm}$ wide, and $23.0 \mathrm{~cm}$ long. Each chamber contained a retractable lever that was situated on one wall of the chamber, $3.0 \mathrm{~cm}$ to the left of the aperture into which $45-\mathrm{mg}$ food pellets could be delivered. When extended, the lever projected $2.2 \mathrm{~cm}$ into the chamber, was $3.8 \mathrm{~cm}$ wide and was located $13.5 \mathrm{~cm}$ from the ceiling and $4.0 \mathrm{~cm}$ from the grid floor. When retracted, the lever 
was flush with the wall of the chamber. When the lever was extended into the chamber, lever contacts were recorded by means of a drinkometer circuit connected to the lever.

The food aperture was $5.0 \mathrm{~cm}$ high and $4.0 \mathrm{~cm}$ wide, and the entrance to this food tray was covered by a Perspex door hinged at the top of the aperture. Pushing the Perspex door activated a microswitch that recorded tray entries. Reinforcement consisted of the delivery of a single $45-\mathrm{mg}$ food pellet and was accompanied by the characteristic "click" of the food hopper and a brief flash of the food tray light. General illumination throughout each experimental session was provided by a small houselight situated on the ceiling of the chamber. The experimental chambers were housed in sound attenuating boxes; the experiment was controlled and data were recorded by solid state logic programming equipment.

\section{Procedure}

Magazine training. On the first day, each subject was placed in the chamber for $30 \mathrm{~min}$ with 10 food pellets present in the food tray. After this adaptation period, each subject was given eight sessions of magazine training in which food pellets were delivered in the food tray on a variable-time (VT) 100 -sec schedule. This schedule had a minimum interval of $15 \mathrm{sec}$ and a maximum interval of $120 \mathrm{sec}$. Each session lasted for 50 food deliveries, and at the end of eight sessions, observation of the animals confirmed that all were taking the pellets from the food tray immediately after delivery.

Training. The subjects were divided into three groups: Both the continuous reinforcement and partial reinforcement groups contained five subjects, and the random control group contained four.

Continuous reinforcement group. For this group, food delivery was now paired with insertions into the chamber of the response lever. These trials occurred with an average intertrial interval of $100 \mathrm{sec}$. The lever was inserted into the chamber $10 \mathrm{sec}$ prior to pellet delivery and was retracted on delivery of the pellet. A session consisted of 50 daily pairings of lever and food.

Partial reinforcement group. This group similarly received 50 lever presentations per day, but only $50 \%$ of these presentations, on a random basis, were followed by food. Thus both continuous and partially reinforced groups received the same number of lever presentations per day, but the partial reinforcement group received only half the number of food deliveries obtained by the continuous reinforcement group.

Random control group. These subjects received 50 lever presentations and 50 food deliveries per session, except that there was no programmed relationship between the two events. Both lever presentations and food deliveries were scheduled on independent VT $100-\mathrm{sec}$ schedules. This training phase lasted for 15 sessions for all groups.

Extinction. Following the training phase, each subject in the continuous and partially reinforced group was given 15 sessions of extinction. These sessions were identical in format and duration to those during acquisition, except that no food was presented at any time during these sessions for either group.

\section{RESULTS}

\section{Sign-Tracking}

Figure 1 illustrates the effects of partial and continuous reinforcement on the acquisition and extinction of lever contacts (sign-tracking). Partially reinforced subjects exhibited a higher overall rate of lever contact across acquisition than continuously reinforced subjects $[F(1,8)=11.304, p<.01]$, and an analysis of variance also revealed a significant Group by Trial interaction $[F(14,112)=1.653, p<.05]$. For the last three sessions of acquisition, rate of lever contact was higher in both the $50 \%$ group $[\mathrm{t}(25)=7.4802, \mathrm{p}<.001]$ and the $100 \%$
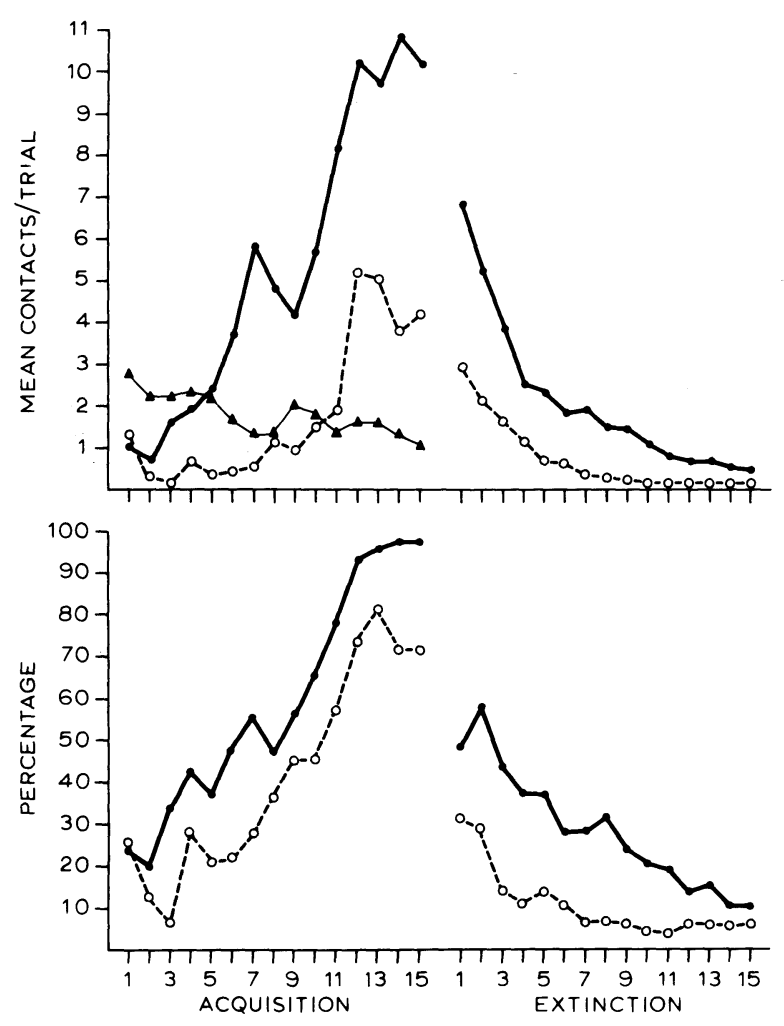

Figure 1. Acquisition and extinction of lever-contact responses for the partially reinforced group (filled circles), continuously reinforced group (open circles), and random control group (triangles). The upper graph shows mean rate of lever contacts during CS presentation, and the lower graph represents the percentage of trials with at least one response.

group $[\mathrm{t}(25)=3.782, \mathrm{p}<.001]$ than in the random control group, suggesting that the lever contact behavior in the two paired groups was not a result of nonassociative factors elevating the operant level of lever contact. A probability measure (bottom left-hand panel of Figure 1) also showed that subjects in the partially reinforced group were responding on a significantly greater percentage of the trial than continuously reinforced subjects were during the last three sessions of acquisition $(\mathrm{U}=54.5, \mathrm{p}<.05)$.

An analysis of variance also indicated that rate of extinction of lever contacts was significantly greater for the partial group, as revealed by a significant Group by Trial interaction $[F(14,112)=3.306, p<.001]$. However, as Mackintosh $(1974$, p. 73$)$ has pointed out, if training has established a higher level of conditioning in one of the groups, then there is already a bias toward obtaining a faster rate of extinction in that group because the higher level of responding provides more room for rate of decline in extinction. One way around this dilemma is to transform the extinction data into scores relative to the asymptotic level obtained by each group at the end of acquisition training. Thus, in the present study, an average rate of lever contacts over the final three sessions of acquisition was calculated for each subject, and the mean for the group is expressed as 
1 on the extreme left of Figure 2. Normalized extinction response functions were then obtained for both groups by dividing each subject's daily response rate by his average rate at the end of acquisition. When this transformation was made, Figure 2 shows that there was no difference in rate of extinction between the

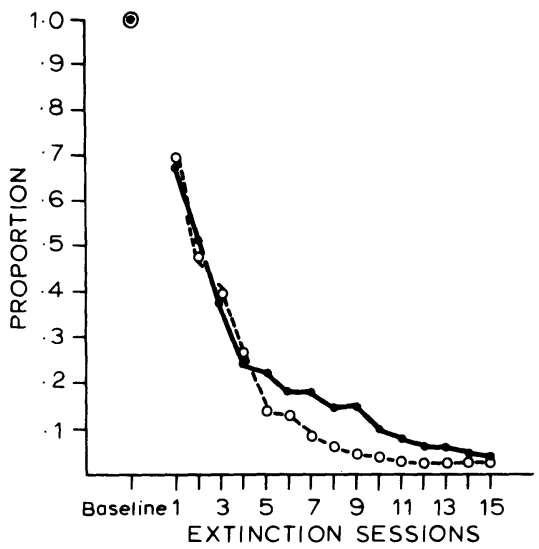

Figure 2. Rate of extinction of the lever contact response as a function of each subject's asymptotic level of lever contact at the end of acquisition. Filled circles represent the partially reinforced group, and open circles represent the continuously reinforced group. See text for further explanation.
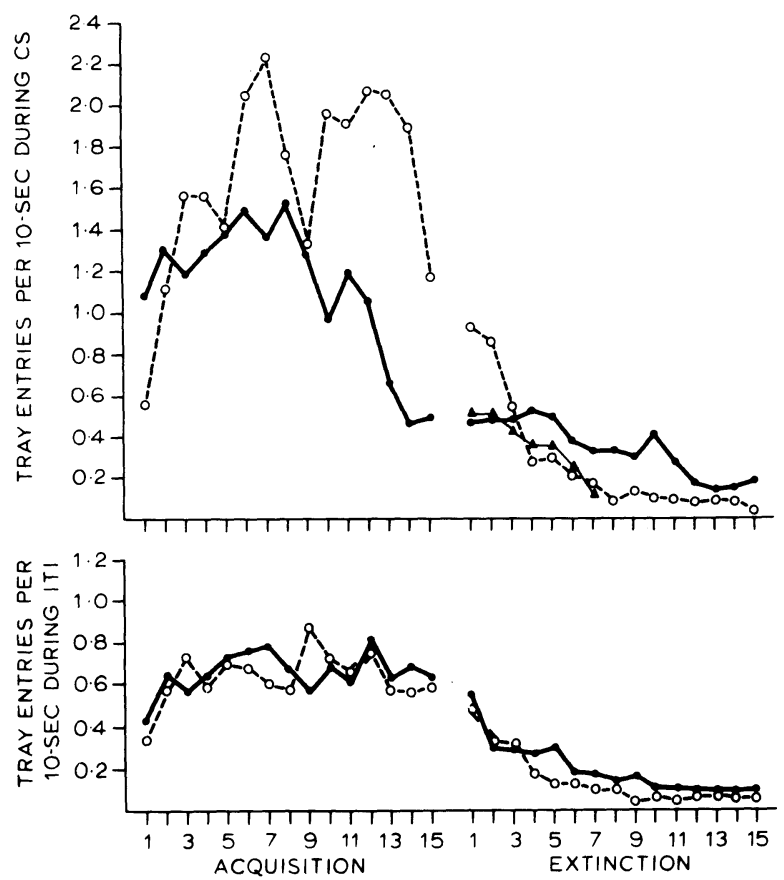

Figure 3. Acquisition and extinction of trayentry responses during CS presentation (top panel) and intertrial interval (bottom panel). Filled circles represent the partially reinforced group, and open circles represent the continuously reinforced group. The triangles in the top panel represent mean tray entry rate per 100 trials of extinction (i.e., two consecutive sessions) for the partially reinforced group. This gives a "perexpectedreinforcer" comparison with the continuously reinforced group. See text for further explanation. partial and continuous groups at least over the first four sessions of extinction. Furthermore, analysis of variance over the full 15 sessions of extinction failed to reveal a significant Group by Trials effect $[F(14,112)=$ .35 , n.s.]. Similarly, no significant Groups by Trials effect was found over extinction when the percentage trials with a response data were put through the same transformation $[F(14,112)=.653$, n.s. $]$.

\section{Goal-Tracking}

Figure 3 shows the rate of tray entry for both groups during both CS presentation and intertrial intervals. Over the final three sessions of acquisition, the continuously reinforced group showed a significantly higher rate of tray entry than the partially reinforced group $(U=25$, $\mathrm{p}<.05)$, but this effect of conditions was not significant over the whole 15 sessions of acquisition $[F(1,8)=$ $2.103, p>.05]$. The bottom left-hand panel of Figure 3 shows no difference in rates of tray entry during the intertrial intervals between groups, suggesting that the differences observed between groups on the duringtrial measure were not simply due to an overall elevated rate of tray entry in the continuous group.

However, during extinction, tray entry in the continuous group shows a significantly faster rate of extinction than in the partial group [Group by Trials $F(14,112)=2.475, p<.01]$. The functions for the two groups also exhibit a crossover, with rate of tray entry being higher in the continuously reinforced group during the first two sessions of extinction $[\mathrm{t}(18)=2.271$, $\mathrm{p}<.05]$ and lower than that for the partially reinforced group in the last two sessions of extinction $[t(18)=2.293, p<.05]$. Even when these data are normalized by transformation into a score relative to their preextinction levels, there is still a significant Group by Trials interaction $[\mathrm{F}(14,112)=2.302, \mathrm{p}<.05]$. Similarly, when a per-reinforcer rather than a per-trial analysis of extinction is made, as some authors recommend (cf. Gibbon et al., 1980), a Group by Reinforcer interaction is still apparent $[\mathrm{F}(6,48)=2.36, \mathrm{p}<.05]$.

\section{DISCUSSION}

The results of this study confirm earlier findings that partial reinforcement facilitates sign-tracking and suppresses goaltracking (Boakes, 1977; Davey, Oakley, \& Cleland, 1981). Furthermore, they also suggest that sign-tracking and goaltracking responses differ in another characteristic: their susceptibility to the PREE. In the light of the robust and highly reliable nature of the PREE found in traditional instrumental studies, this result makes it difficult to conceive of the signtracking observed in this study as being controlled solely by instrumental factors.

However, discovering that goal-tracking does exhibit the PREE does not automatically make it a candidate for an instrumental response. Alternatively, rather than considering the difference in PREE susceptibility as evidence for or against instrumental control per se, the differential PREE found in the present study could reflect the effects of partial reinforcement on a chain or sequence of behaviors. For instance, Mellgren (cited in Morgan, 1979) found that responses early in an appetitive response chain failed to exhibit the PREE, whereas those that terminated the chain and were proximal to reinforcer 
delivery did exhibit the PREE. In other studies, we have found that sign-tracking is more likely to occur in the first half of the trial and goal-tracking is more probable in the second half of the trial (Davey et al., 1981; see also Holland, 1977). If the autoshaping episode consists of a response sequence consisting of signal approach followed by food-tray approach, we might expect that the later components (goal-tracking) would exhibit the PREE and the early components (sign-tracking) would not. This kind of analysis is consistent with the results of Gibbon et al. (1980) using pigeons. At first glance, their results might seem to contradict those of the present experiment: They found evidence for a PREE using the pigeon's autoshaped keypeck. However, pigeons are a species that fails to exhibit overt signs of goal-tracking in the prototypical autoshaping situation (Boakes, 1979) and spend almost the entire CS duration sign-tracking. If the PREE is a phenomenon that afflicts only those responses that regularly occur in close temporal proximity to reinforcer delivery, a PREE in sign-tracking with pigeons would be predicted. But this prediction would be true of rats only if goaltracking was eliminated or significantly suppressed.

Nevertheless, these results indicate that although goal-tracking and sign-tracking in the rat are both discriminated responses emitted in the presence of the food-related CS, they possess a number of different dynamic characteristics. Their frequency of occurrence is differentially affected by partial reinforcement, and one response (goal-tracking) exhibits the PREE, whereas the other (sign-tracking) does not.

\section{REFERENCES}

Atrip, G. W. Stimulus and response-reinforcer contingencies in autoshaping, operant, classical and omission training procedures in rats. Journal of the Experimental Analysis of Behavior, 1977, 28, 59-69.

Bonkes, R. A. Performance on learning to associate a stimulus with positive reinforcement. In H. Davies \& H. M. B. Hurwitz (Eds.), Operant-Pavlovian interactions. Hillsdale, N.J: Erlbaum, 1977.

Bonkes, R. A. Interaction between Type I and Type II processes involving positive reinforcement. In A. Dickinson \& $\mathbf{R}$. A. Boakes (Eds.), Mechanisms of learning and motivation. Hillsdale, N.J: Erlbaum, 1979.

Davey, G. C. L., OAkley, D., \& Cleland, G. G. Autoshaping in the rat: Effect of omission on the form of response. Journal of the Experimental Analysis of Behavior, 1981, 36, 75-91.

Farwell, B. J., \& AYres, J. J. B. Stimulus-reinforcer and response-reinforcer relations in the control of conditioned appetitive headpoking ("goal-tracking") in rats. Learning and Motivation, 1979, 10, 295-312.

Gamzu, E., \& Williams, D. R. Classical conditioning of a complex skeletal response. Science, 1971, 171, 923-925.

Gamzu, E., \& Williams, D. R. Associative factors underlying the pigeon's key-pecking in autoshaping procedures. Journal of the Experimental Analysis of Behavior, 1973, 19, 225-232.

Gibbon, J., Baldock, M. D., Locurto, C., Gold, L., \& Terrace, H. Trial and intertrial durations in autoshaping. Journal of Experimental Psychology: Animal Behavior Processes, 1977, 3, 264-284.

Gibbon, J., Farrell, L., Locurto, C. M., Duncan, H. J., \& Terrace, H. S. Partial reinforcement in autoshaping. Animal Learning \& Behavior, 1980, 8, 45-59.

Hearst, E. Classical conditioning as the formation of interstimulus associations: Stimulus substitution, parasitic reinforcement and autoshaping. In A. Dickinson \& R. A. Boakes (Eds.), Mechanisms of learning and motivation. Hillsdale, N.J: Erlbaum, 1979.

Holland, P. C. Conditioned stimulus as a determinant of the form of the Pavlovian conditioned response. Journal of Experimental Psychology: Animal Behavior Processes, 1977, 3, 77-104.

LEwis, D. J. Partial reinforcement: A selective view of the literature since 1950. Psychological Bulletin, 1960, 57, 1-29.

MACKInTOSH, N. J. The psychology of animal learning. London: Academic Press, 1974.

Morann, M. J. Motivational processes. In A. Dickinson \& R. A. Boakes (Eds.), Mechanisms of learning and motivation. Hillsdale, N.J: Erlbaum, 1979.

Schwartz, B., \& Williams, D. R. The role of the responsereinforcer contingency in negative automaintenance. Journal of the Experimental Analysis of Behavior, 1972, 17, 35-37.

Terrace, H. S., Gibbon, J., Farrell, L., \& Baldock, M. D. Temporal factors influencing the acquisition and maintenance of an autoshaped key-peck. Animal Learning \& Behavior, 1975, 3, 53-62.

WeARDEN, J. H. Omission training of signalled approach to the site of food delivery in rats. Behaviour Analysis Letters, in press.

Williams, D. R., \& Williams, H. Automaintenance in the pigeon: Sustained pecking despite contingent nonreinforcement. Journal of the Experimental Analysis of Behavior, 1969, 12, 511-520.

(Received for publication November 13, 1981.) 